\title{
Some Aspects of the Flow-Topography Interactions in the Taiwan Strait
}

\author{
Ching-Sheng Chern ${ }^{1, *}$ and Joe Wang ${ }^{1}$ \\ (Manuscript received 4 March 2000, in final form 28 June 2000)
}

\begin{abstract}
The Taiwan Strait is a shallow channel with complicated topographic variations that connects the South China Sea with the East China Sea. Previous hydrographic and numerical studies have suggested that a distinct feature of the flow pattern in the strait is the transfer of the vertical stratification of the incoming flow from the south into the horizontal density gradient in the middle of the northern portion of the strait. This work applies a numerical model with a free surface to examine the influence of a change in the volume transport on the density distribution in the strait. According to the model results, a reduction in the incoming flow rate will cause a weakening of the original density gradient. The release of the available potential energy associated with the original density gradient causes the current to meander. The model results are consistent with the satellite sea surface temperature images on 12 and 17 August, 1998.
\end{abstract}

(Keywords: Taiwan Strait, Numerical model, Available potential energy)

\section{INTRODUCTION}

The Taiwan Strait (TS) is a major channel for the exchange of water masses between the East China Sea and the South China Sea (SCS). Although the mean water depth of the TS is around $60 \mathrm{~m}$, there is considerable topographic variation. Figure 1 depicts the bathymetry in the vicinity of the TS. The main features include the shallow Formosa Bank, the Peng-hu Channel (PHC), the Chang-yuen Ridge (CYR) and the Kuan-Yin Depression (KYD).

During summer, the southwesterlies drive the SCS waters northward. Some of them pass through the PHC and enter the TS (Fan and Yu 1981). Jan et al. (1994) used a numerical model to study the distribution of the SCS water in the TS. They found that the stratified SCS water entering the PHC veers in front of the CYR. The warm surface water can flow over the CYR and hug the west coast of Taiwan, but the cold deep water is deflected westward and occupies

\footnotetext{
${ }^{1}$ Institute of Oceanography, National Taiwan University, Taipei, Taiwan, ROC

* Corresponding author address: Prof. Ching-Sheng Chern, Institute of Oceanography, National Taiwan University, \#1, section 4, Roosevelt Rd., Taipei, Taiwan, ROC; E-mail: chern@ ipx.oc.ntu.edu.tw
} 


\section{Bottom Topography (km)}

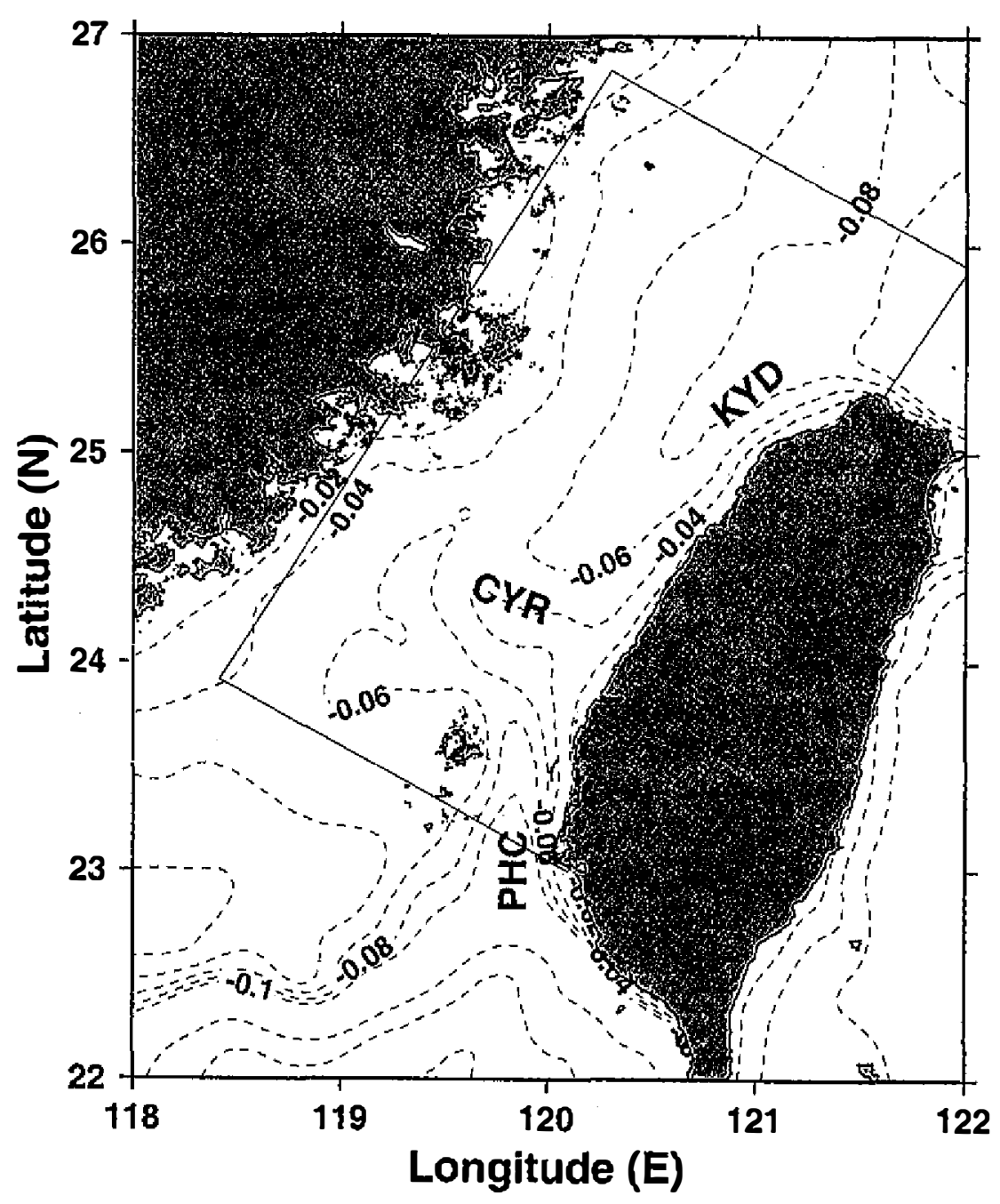

Fig. 1. The bottom topography of the Taiwan Strait. The depth contours are in km, PHC denotes the Peng-hu Channel, CYR denotes the Chang-yuen Ridge and KYD denotes the Kuan-yin Depression. The rectangle inside the figure is our model region.

the western portion of the TS. Therefore, the vertical stratification in the PHC is transferred into the cross-strait density gradient north of the CYR. Most of the hydrographic surveys in the TS during the summer have shown that the temperature and salinity contours in the northern part of the TS are roughly parallel to the coast (Wang and Chern 1992).

However, as the northward transport in the TS becomes weak, corresponding to a period 
of calm southwesterly wind, flow in the TS is no longer confined to the direction parallel to the coast. Some water begins to move perpendicular to the direction of the strait (Chern and Wang 1992).

In this paper, a three-dimensional primitive equation model with a free surface is used to study the flow adjustment in the TS with the occurrence of the water motion across strait. We find that the decrease in the incoming transport causes the weakening of the cross-strait density gradient, and the release of the available potential energy causes the current to meander when the stratification of the incoming water in the $\mathrm{PHC}$ is sufficiently strong.

\section{NUMERICAL MODEL}

The basic equations for the model closely follow those in Semtner (1986). Under the Boussinesq and hydrostatic approximations, the model equations are as follows.

$$
\begin{aligned}
\frac{D u}{D t}-f v & =-\frac{1}{\rho_{0}} P_{x}+A_{m} \Delta u+v u_{z z}, \\
\frac{D v}{D t}+f u & =-\frac{1}{\rho_{0}} P_{y}+A_{m} \Delta v+v v_{z z}, \\
\frac{\partial P}{\partial z} & =-g \rho, \\
u_{x}+v_{y}+w_{z} & =0, \\
\frac{D T}{D t} & =A_{h} \Delta T+\kappa T_{z z}, \\
\frac{D S}{D t} & =A_{h} \Delta S+\kappa S_{z z}, \\
\rho & =\rho(S, T, P),
\end{aligned}
$$

where $(\mathrm{u}, \mathrm{v}, \mathrm{w})$ are velocities in the $(\mathrm{x}, \mathrm{y}, \mathrm{z})$ directions, $\rho$ is the density, $\rho_{0}$ is a reference water density, $\mathrm{f}$ is the Coriolis parameter, $\mathrm{T}$ is the temperature, $\mathrm{S}$ is the salinity, $\mathrm{P}$ is the pressure, $\left(A_{m}\right.$, $\left.A_{h}\right)$ are the horizontal mixing coefficients for momentum and temperature/salinity, respectively, $v$ is the vertical viscosity for momentum, $\kappa$ is the vertical diffusivity,

$$
\frac{D}{D t}=\frac{\partial}{\partial t}+u \frac{\partial}{\partial x}+v \frac{\partial}{\partial y}+w \frac{\partial}{\partial z} \text { and } \Delta=\frac{\partial^{2}}{\partial x^{2}}+\frac{\partial^{2}}{\partial y^{2}} \text {. }
$$

The method of integration retains the split into barotropic and baroclinic modes. We define $u=\bar{u}+u^{\prime}$ and $v=\bar{v}+v^{\prime}$, where $(\bar{u}, \bar{v})$ is the vertically averaged barotropic mode velocity and $\left(u^{\prime}, v^{\prime}\right)$ is the baroclinic internal mode flow with no depth average. We still use the Semtner algorithm to compute the $\left(u^{\prime}, v^{\prime}\right)$ velocity and the (T,S) fields. However, a code similar to that of the Princeton Ocean Model (POM) developed by Blumberg and Mellor (1987) 
is adopted to compute the barotropic mode motion. Our approach is also very similar to the model in Chao and Paluszkiewicz (1991).

The model domain, as shown by the rectangle in Fig. 1, has a 40x75 grids in the horizontal plane. The mesh size is $5 \mathrm{~km}$ in both the $\mathrm{x}$ and $\mathrm{y}$ directions, where the $\mathrm{x}$-axis is transverse to the strait and the $y$-axis is along the strait. There are ten levels in the vertical with a layer thickness of $10 \mathrm{~m}$. The two sides along the strait of the model basin are regarded as rigid walls and the other two as open boundaries. Figure 2 shows the model basin and includes all the main topographic features shown in Fig. 1. The Peng-hu Islands and the Hai-tan Islands are treated as a single bigger island in the model basin.

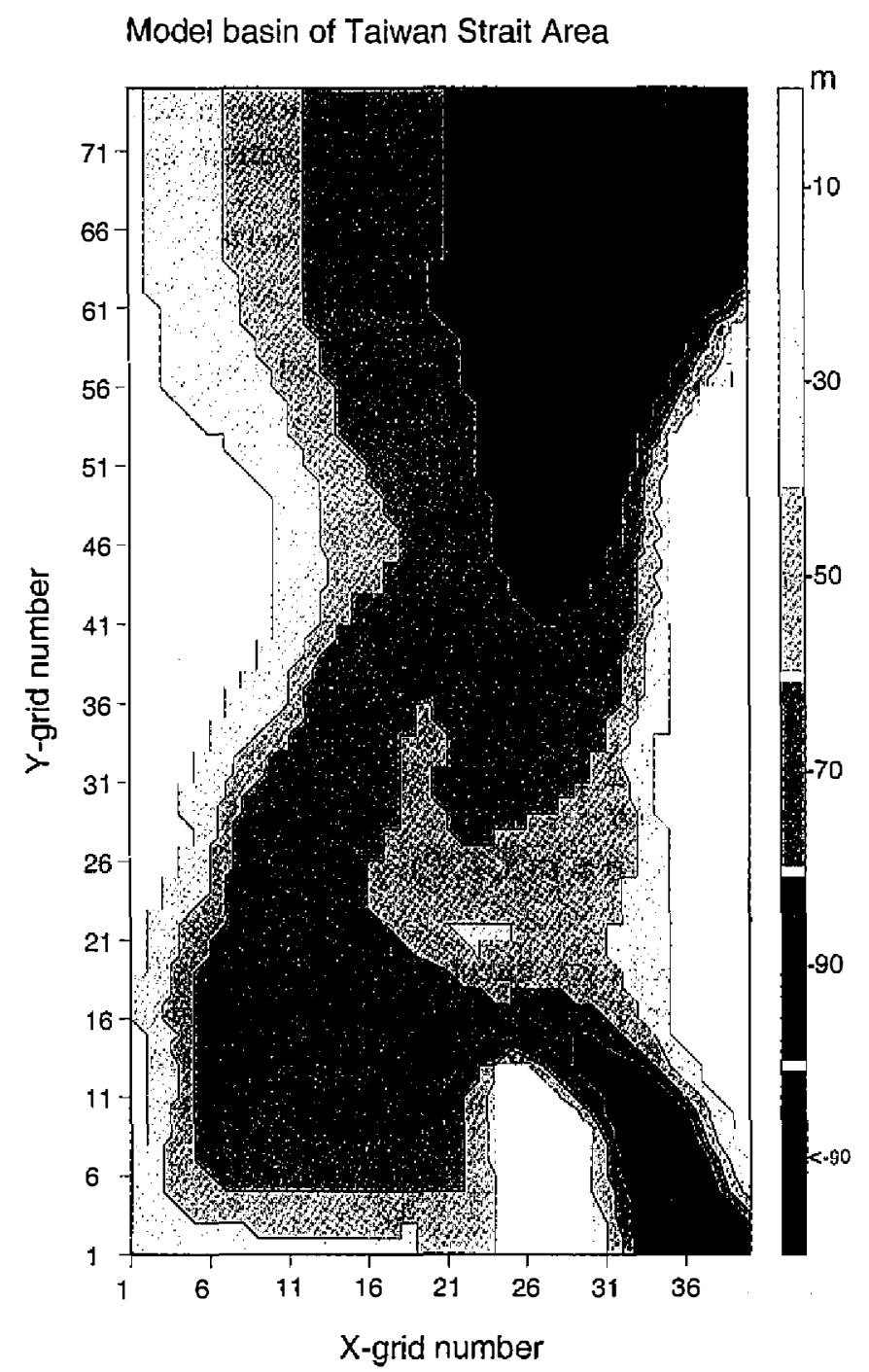

Fig. 2. Topography of the model basin; the annotations are grid numbers in the horizontal axis and the depths are in meters. 


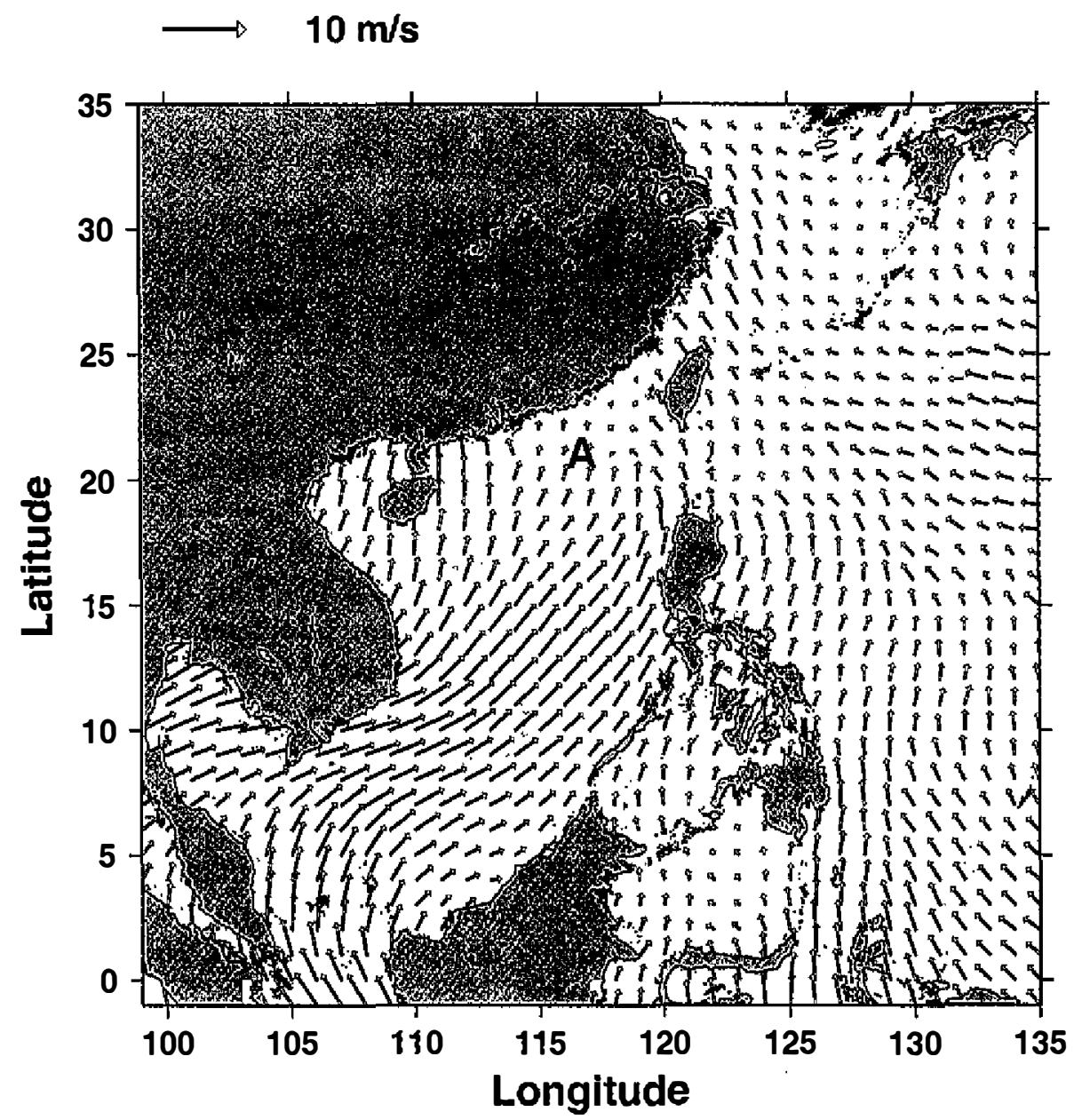

Fig. 3. The surface wind distribution, average of July and August 1996, over the seas surrounding Taiwan area. Sta. A denotes Tungsha Island.

The wind field in the region around Taiwan is dominated by the seasonal monsoon changes. Figure 3 depicts the average surface wind distribution over the SCS and TS area, as calculated from the satellite SSM/I wind data during July and August 1996. We inferred that during summertime, the winds in the TS are generally weaker than those in the SCS. Therefore, the summertime flow pattern in the TS is mainly influenced by the magnitude of the northward transport, which enters the TS through the PHC. The variation of the flow rate in the PHC certainly depends on the wind strength in the SCS. Jan et al. (1994) estimated that the volume ransport is about $1 \mathrm{~Sv}$ in the PHC during summer, and the hydrographic field calculated from a rigid lid model based on this flow rate is very close to that from the observed data. In the following model studies, we also used this transport value as a standard case.

Initially, there is no motion in the model basin and the water has a constant temperature and salinity, $\left(24^{\circ} \mathrm{C}, 34 \mathrm{psu}\right)$. The model ocean is driven with a volume transport of $1 \mathrm{~Sv}$ 
flowing in from the PHC. The velocity of the inflow at the PHC has no vertical variation and its horizontal profile is assumed to have a parobolic shape, given by

$$
v(x)=\frac{6 Q}{D h^{3}}[x(h-x)],
$$

where $Q$ is the flow rate, $(D, h)$ are the depth and width of the PHC, chosen to be $(100 \mathrm{~m}, 50$ $\mathrm{km}$ ), and $x$ is the distance measured from the lower right comer of the model basin. At the open boundary to the west of Peng-hu and at the north of the model domain, we use the Orlanski explicit radiation condition for the water level and velocity fields. The no-slip condition is used at all rigid boundaries.

The water to the south of the PHC has properties which vary between spring and summer. Wang and Chern (1988) showed that water originating in the Kuroshio, the so-called "Kuroshio branch current", enters the TS only during the spring. After the onset of the southwesterly monsoon, the SCS water will be present in the TS. Therefore, we choose two different temperature and salinity profiles, as shown in Fig. 4, for the water flowing into the PHC. These two profiles were observed at the entrance of the PHC on 2 September and 8 June, 1988 respectively, and can be regarded as representing the waters from the SCS, Case I, and the Kuroshio branch current, Case II. The SCS water has a stronger thermocline and less saline surface water, while the water from the Kuroshio branch current has a weaker vertical stratification. We use the no-normal-gradient condition for the temperature and salinity fields at the other open boundaries. The no-flux condition for the temperature and salinity is imposed at the sea surface and the rigid boundaries. The horizontal mixing coefficients for the momentum and temperature/salinity balance equations, $\left(A_{m}, A_{h}\right)$ are $4 \cdot 10^{6} \mathrm{~cm}^{2} / \mathrm{s}$ and $2 \cdot 10^{6} \mathrm{~cm}^{2} / \mathrm{s}$, respectively. The vertical mixing coefficients, $v$ and $\kappa$, are $5 \mathrm{~cm}^{2} / \mathrm{s}$ for all the momentum, temperature and salinity fields.

The numerical model was integrated for 400 days. Figure 5 shows the time variations of the kinetic energy, temperature and salinity, averaged over the whole model basin, for Case I. This figure indicates that the flow reaches a quasi-steady state after 250 days.

Figure 6 depicts the horizontal distribution of the temperature, salinity and velocity fields at $15 \mathrm{~m}$ and $45 \mathrm{~m}$ for Case I on day 250. The temperature and salinity distribution of Fig. 6 are similar to the observed pattern of the hydrographic survey in the TS during 1 to 6 September 1988 (Fig. 3 in Wang and Chern 1992). Due to the blocking of the CYR, the cold and saline water in the lower half of the PHC is deflected westward. Some of this cold $\left(<27^{\circ} \mathrm{C}\right)$ and saline ( $>33.6 \mathrm{psu}$ ) water flows out of the model domain through the open boundary to the west of Peng-hu, while the rest enters the western part of the strait north of the CYR. Transfer of the vertical stratification in the PHC into the cross-strait temperature/salinity gradients north of the CYR is evident. The flow pattern revealed in Fig. 6 resembles the results from Jan et al. (1994) and can be regarded as a typical case of summertime circulation in the TS. In the following studies, we use this pattem as a new initial condition and investigate the response in the TS when the volume transport through the PHC is changed. Since the salinity pattern in Fig. 6 is almost the same as that of the temperature distributions, low salinity is always associated with high temperature. Thus, we will only show the temperature and velocity fields in the following discussions. 

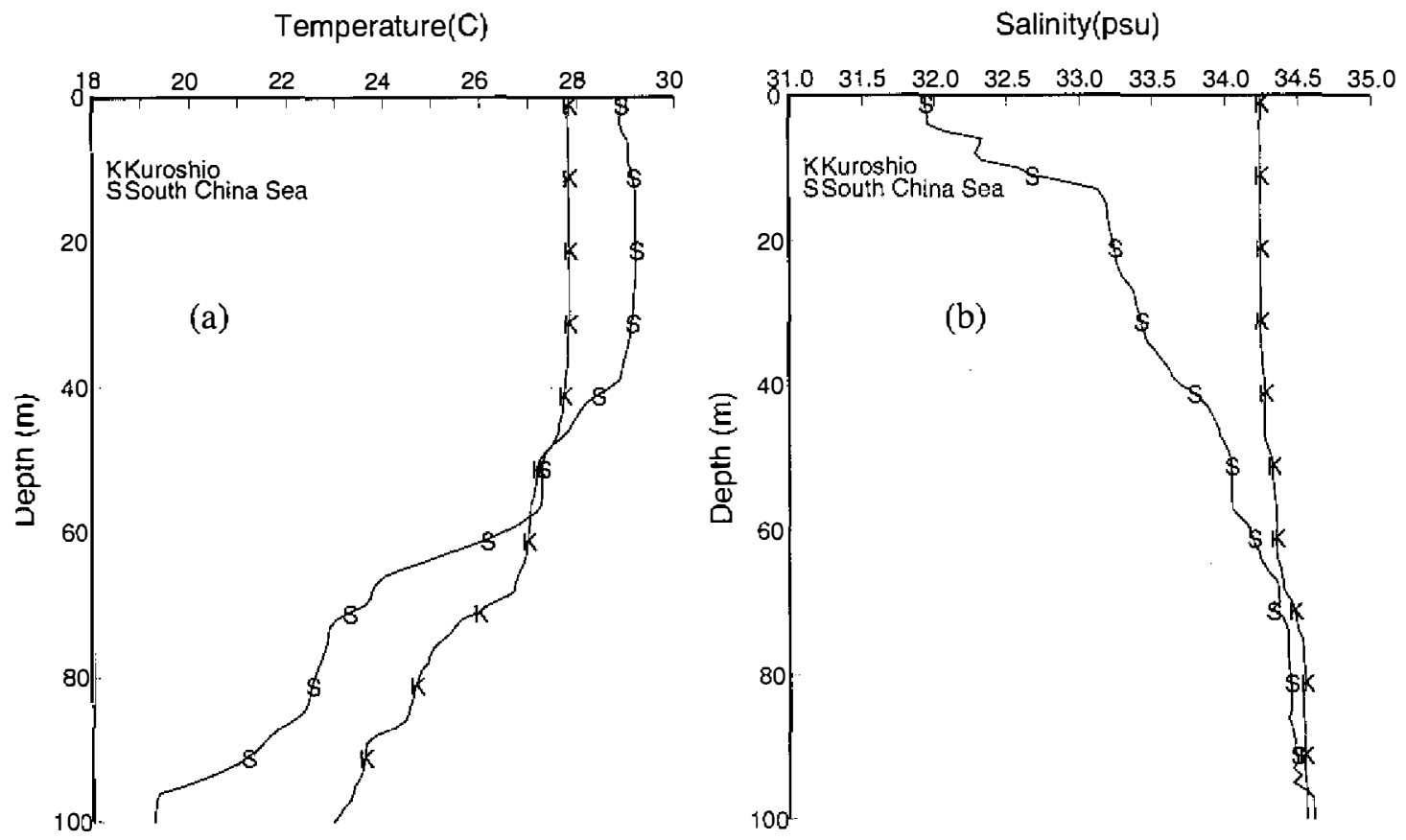

Fig. 4. Typical vertical profiles of temperature (a) and salinity (b) of the SCS water and Kuroshio Branch current at the entrance of PHC.

\section{MODEL RESULTS}

As mentioned in the previous section, the wind over the TS is not strong during the summer. The variation of the flow in the TS depends on the change of the volume transport in the PHC, which in turn depends on the wind variations over the South China Sea. Therefore we may expect a typical time scale for the velocity variation in the PHC to be of the order of a week to a month. In the following, we simulate a case, the Case I-1, in which the result shown in Fig. 6 is used as a new initial condition and reduces the transport in the PHC to $0.5 \mathrm{~Sv}$.

As the velocity of the inflow in the PHC is reduced, the surface warm water can still flow over the CYR and enter the TS, but its ability to uplift the cold lower layer water in the PHC and transport it to the westem portion of the strait will be decreased. Hence the strength of the density gradient to the north of the CYR cannot be maintained. Some adjustment of the flow in the TS is activated. We integrate this model for 100 days. Although a final quasi-steady state, which has a much weaker temperature/salinity gradients north of the CYR, can be reached, this final state is not relevant to the actual condition. We are only interested in the flow adjustment, in which the original frontal strength is retained. The model results show that this kind of flow pattern is present for about 10 days after the reduction of the transport in the PHC and the flow remains similar for up to 30 days of integration. Therefore, in this section, we only discuss the result on day 20 . 


\section{Temperature}

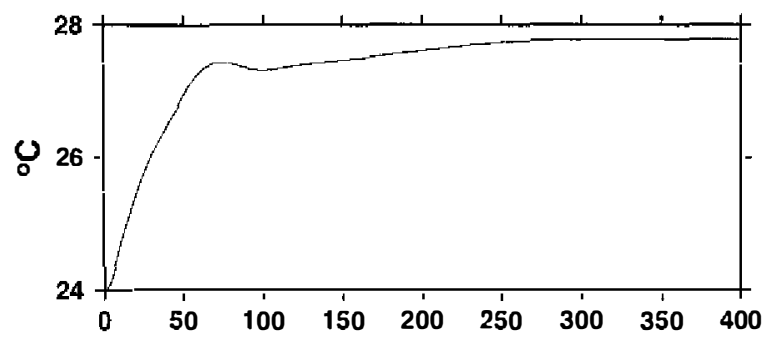

Salinity

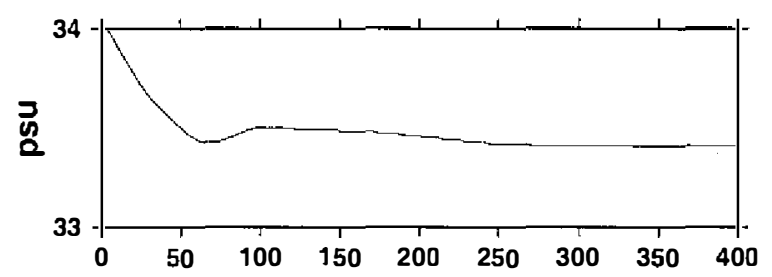

Kinetic Energy

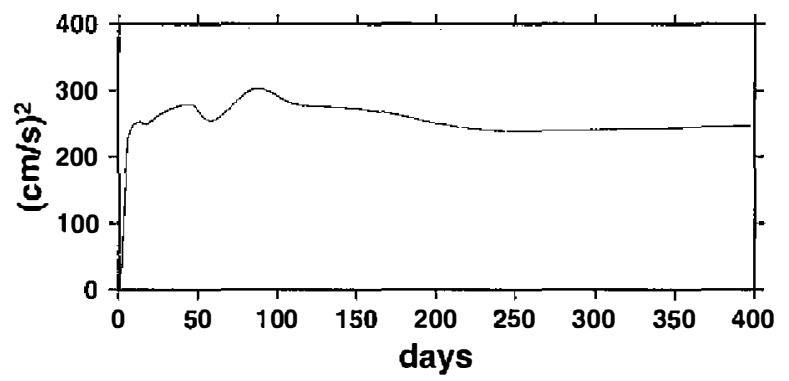

Fig. 5. Time variations of the averaged kinetic energy, temperature and salinity over the whole model basin of the Case I.

Figure 7 depicts the horizontal distribution of the temperature and velocity fields at $15 \mathrm{~m}$ and $45 \mathrm{~m}$ on day 20 , after a reduction of the sonsport in the PHC to $0.5 \mathrm{~Sv}$. The temperature and salinity values of the inflowing water remain the same as those in Case I. Some water (> $27^{\circ} \mathrm{C}$ ) present in the TS north of CYR in Case I, will flow out the TS through the open boundary to the west of Peng-hu in this case. Thus, as it encountered the CYR, the warm water $\left(>27^{\circ} \mathrm{C}\right)$ in Case I-1 spreads over a larger area than in Case I. As a result, during the adjustment process, the velocity of the warm water in the eastern part of the strait drops more than the corresponding value of the cold water in the western part.

Figure 8 shows a cross-strait plot, at grid $Y=35$, of the downseam velocity component at $15 \mathrm{~m}$ for Case I and I-1. The region of higher velocity has moved from the eastern portion (between X-grid number 25 and 34) in Case I, toward the middle portion of the strait (between $\mathrm{X}$-grid number 15 and 25) in Case I-1. This is a general feature of the velocity distribution in the TS north of the CYR as shown in Figs. 6 (a) and 7 (a). Due to its low velocity, the flow in 


\section{Temperature}

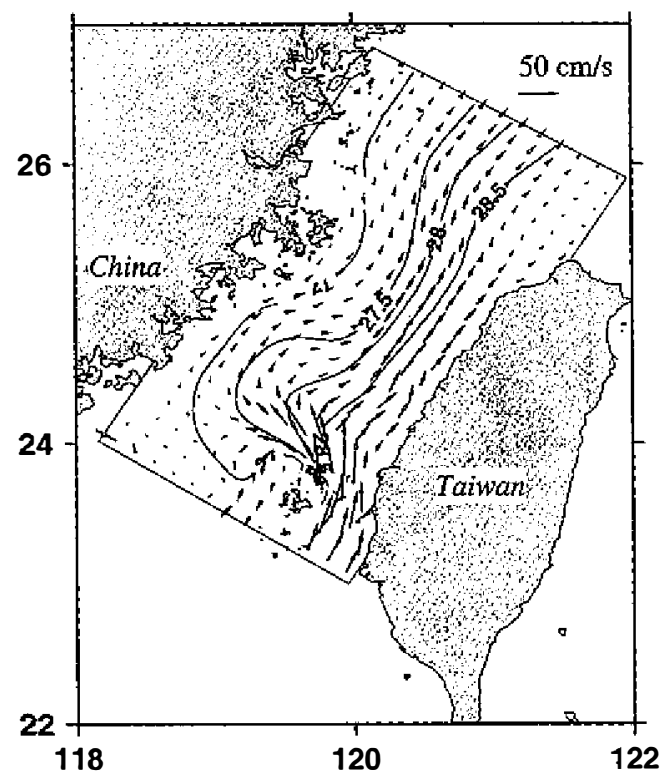

Temperature

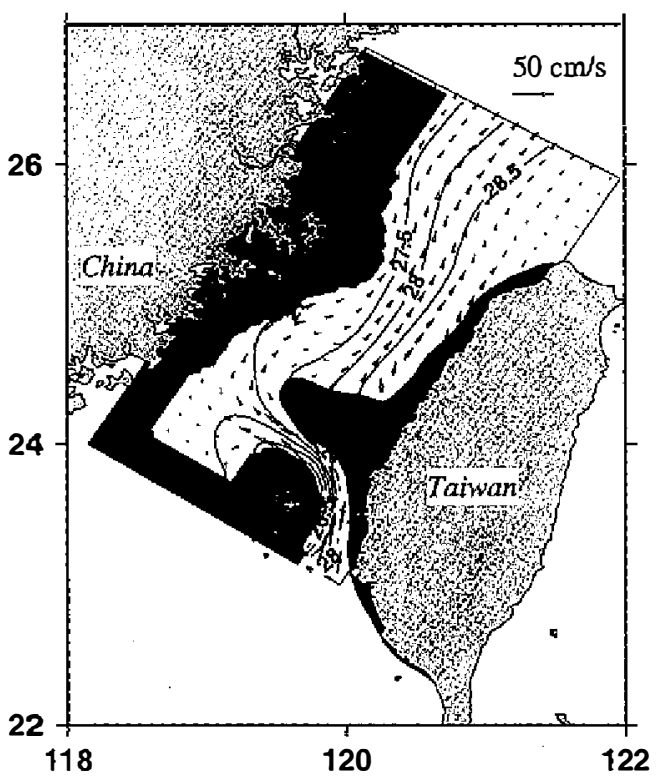

(a)

\section{Salinity}

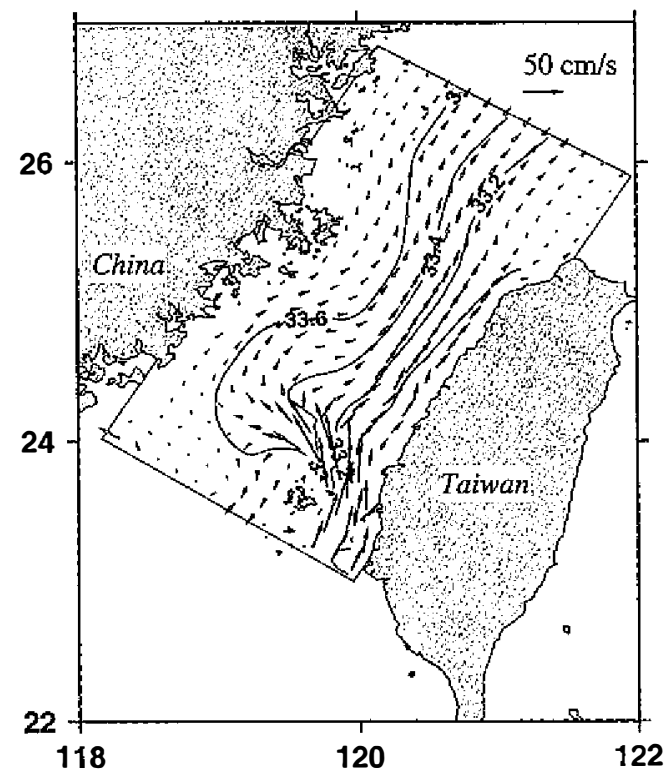

(b)

\section{Salinity}

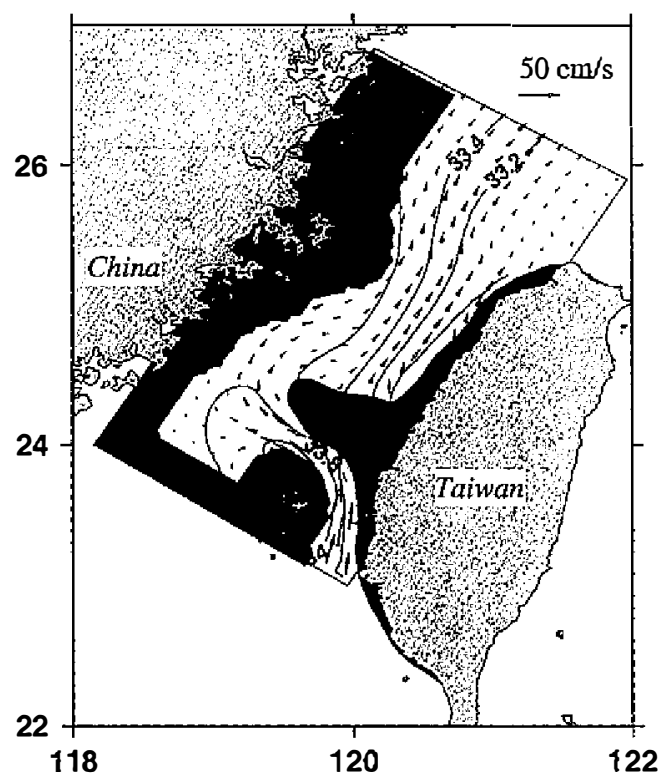

Fig. 6. Horizontal temperature, salinity and velocity distributions at $15 \mathrm{~m}$ (a) and $45 \mathrm{~m}$ (b) for Case I on day 250. 
Case I-1 (15 m)

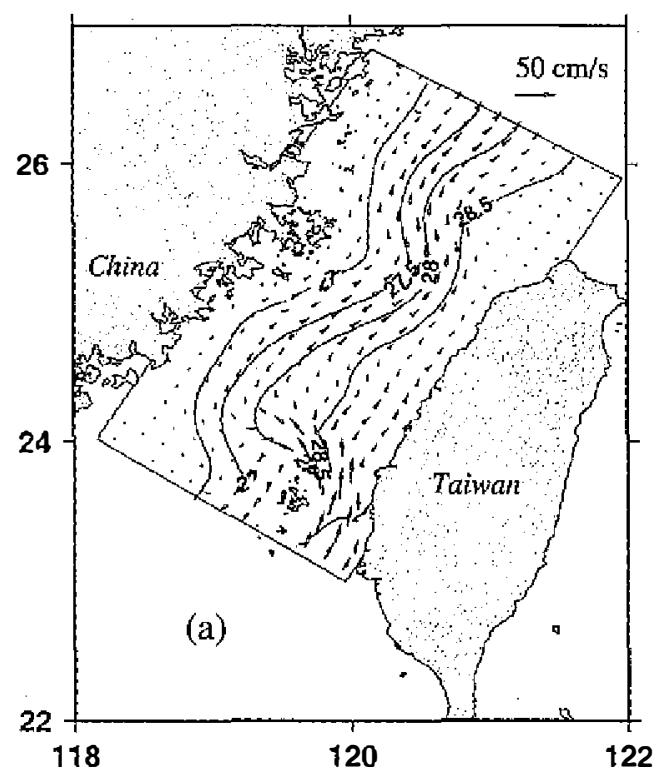

Case I-1 (45 m)

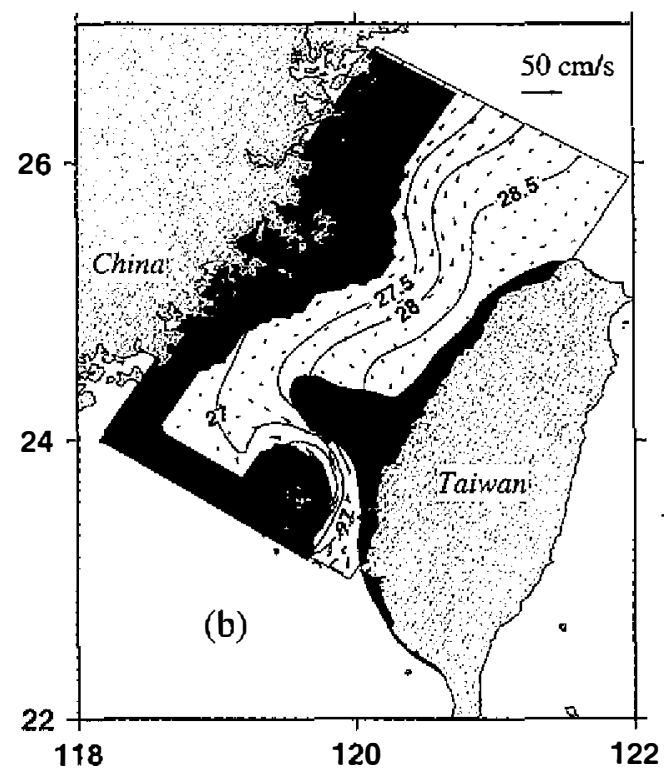

Fig. 7. Horizontal temperature and velocity distributions at $15 \mathrm{~m}$ (a) and $45 \mathrm{~m}$ (b) for Case I-1 on day 20.

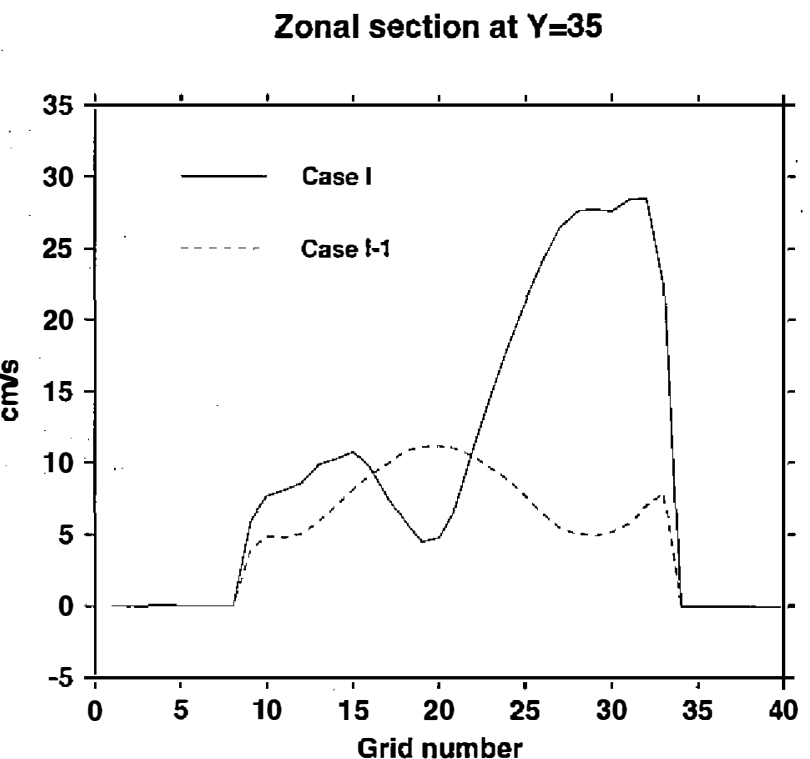

Fig. 8. Cross-strait plot of the downstream velocity at zonal section $\mathrm{Y}=35$ for Case I and I-1. 


\section{Case II (15 m)}

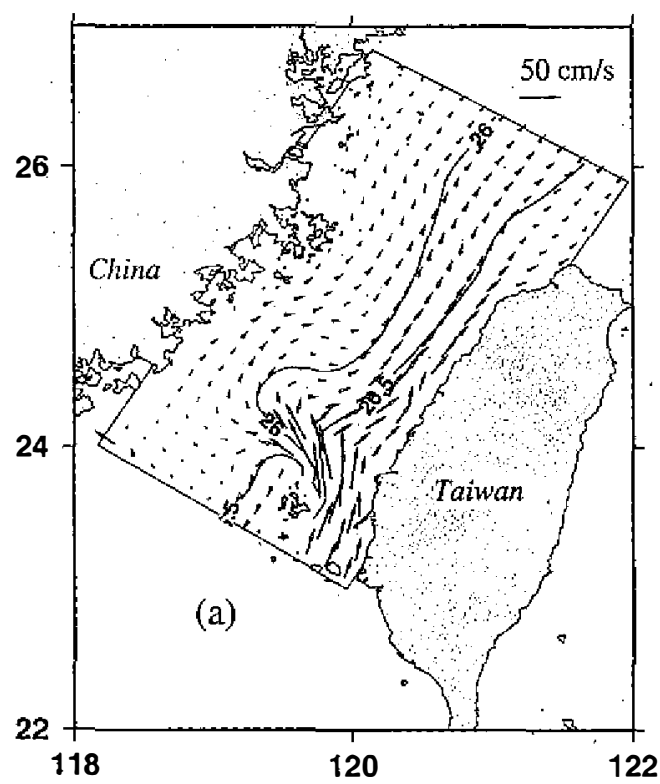

Case II (45 m)

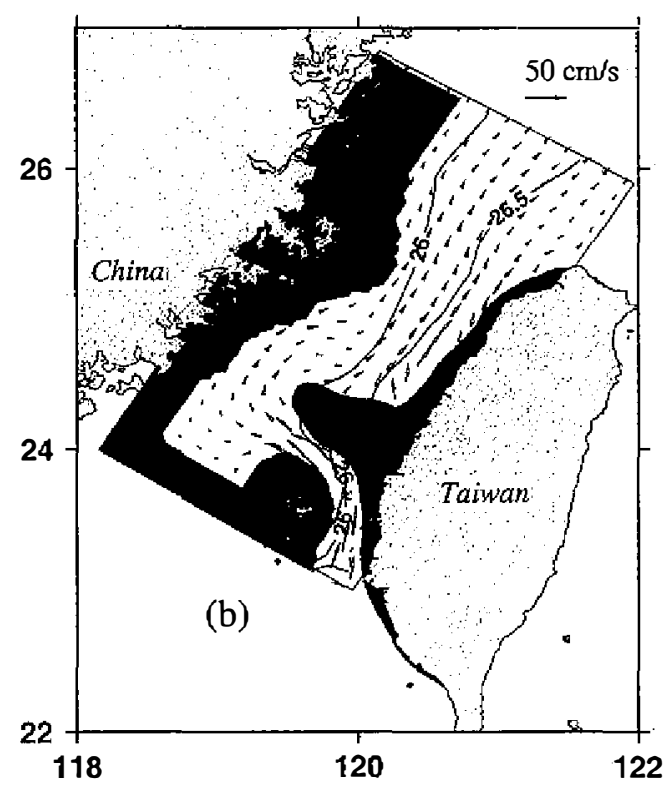

Fig. 9. Horizontal temperature and velocity distributions at $15 \mathrm{~m} \mathrm{(a)} \mathrm{and} 45 \mathrm{~m}$ (b) for Case II on day 250.

the northern TS follows the local topography more closely and the warm water tends to seperate from the right bank of the strait as it encounters the KYD in Case I-1.

Figure 9 shows the horizontal temperature and velocity distributions at $15 \mathrm{~m}$ and $45 \mathrm{~m}$ on day 250 for Case II, in which the inflowing water in the PHC has a weaker stratification. The flow rate in the PHC remains $1 \mathrm{~Sv}$. The transfer of vertical stratification in the PHC into the horizontal density gradient north of the CYR still occurs, but the gradient strength becomes much weaker.

Figure 10 depicts the horizontal temperature and velocity distributions at $15 \mathrm{~m}$ and $45 \mathrm{~m}$ on day 20 of the flow, which uses the result from Case II as a new initial condition and reduces the volume transport at the PHC to $0.5 \mathrm{~Sv}$. Since the vertical stratification of the inflow in the PHC is much weaker in Case II and II-1, the influence of the density structure on the flow in the TS becomes less important. The flow in the strait should depend mainly on the incoming flow rate in the PHC and the topographic variations. The Rossby numbers, based on the velocity of the incoming flow and the width of the PHC, are 0.1 and 0.05 for Case II and II- 1 , respectively, and can be regarded as small. Therefore we may expect that the flow pattern for Case II-1 is similar to that of Case II. The region of higher velocity occurs in the eastern portion of the strait in these two cases. Figure 11 depicts the cross-srait plot, at grid $Y=35$, of the downstream velocity component at $15 \mathrm{~m}$ for Case II and II-1. The similarity of the velocity profiles in these two cases is evident.

Figure 12 depicts the velocity and the vertical component of the relative vorticity distribu- 


\section{Case II-1 (15 m)}

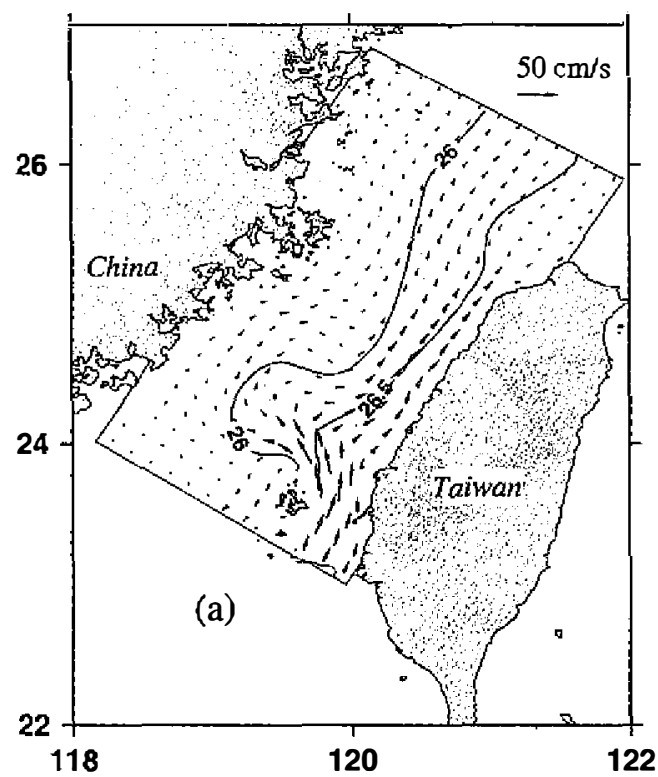

Case II-1 (45 m)

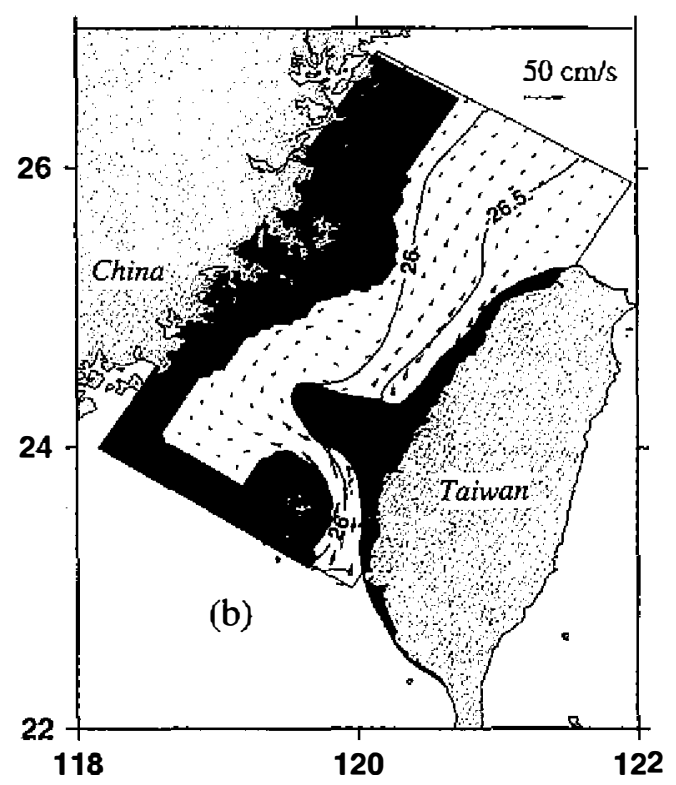

Fig. 10. Horizontal temperature and velocity distributions at $15 \mathrm{~m}$ (a) and $45 \mathrm{~m}$ (b) for Case II-1 on day 20.

\section{Zonal section at $\mathrm{Y}=35$}

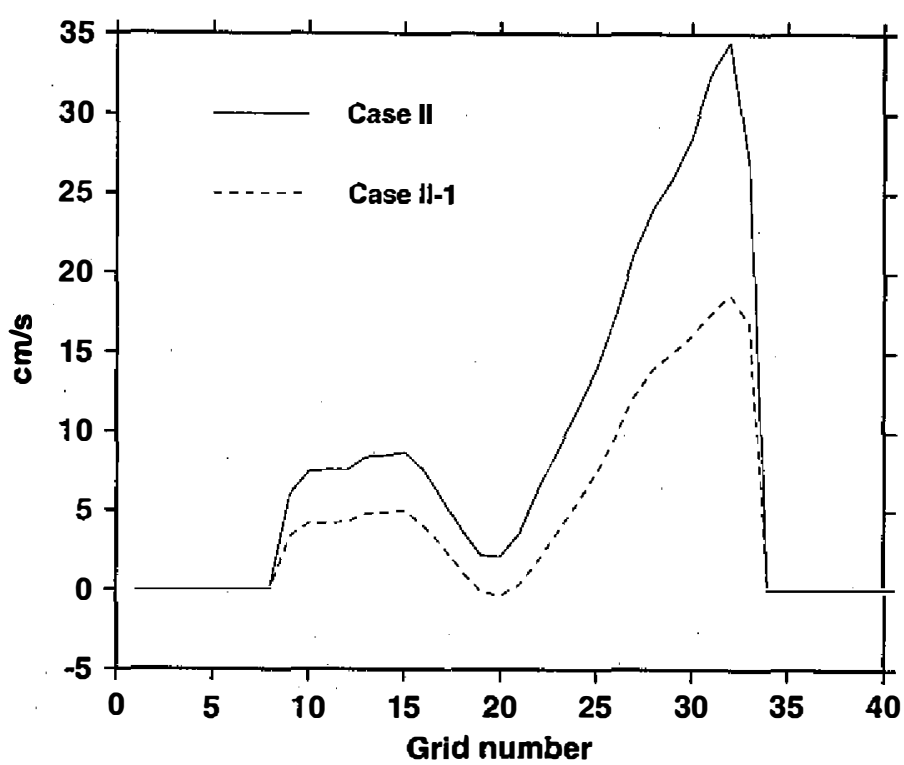

Fig. 11. Cross-strait plot of the downstream velocity at zonal section $Y=35$ for Case II and II-1. 
tions at $15 \mathrm{~m}$ for Case I and II on day 250 . The horizontal friction generates a negative vorticity region along the eastern bank of the strait and the topographic effects cause a negative vorticity region above the CYR. As the flow passes through the CYR, its vertical stratification becomes very weak. The warm water to the east of the temperature front can be regarded as a homogeneous fluid. Therefore the vorticity balance of this warm water can be approximated by the following equation:

$$
\frac{d}{d t}\left(\frac{f+\zeta}{h}\right)=-\frac{1}{h} \vec{\kappa} \cdot \operatorname{curl}\left(\frac{\vec{\tau}}{\rho h}\right)
$$

where $\zeta$ is the relative vorticity, defined as $\left(\frac{\partial v}{\partial x}-\frac{\partial u}{\partial y}\right), \mathrm{h}$ is the water depth, $\vec{\kappa}$ is the unit vector in the vertical direction and $\vec{\tau}$ is the bottom drag. The use of a quadratic drag law, $\vec{\tau}=C \rho|\vec{u}| \vec{u}$ yields that the damping term in the vorticity equation is of the order $R \cdot \frac{\zeta}{h}$, where $R=\frac{C|\vec{u}|}{h}$. The typical values of $|\vec{u}|$ and $\mathrm{h}$ around the CYR are about $25 \mathrm{~cm} / \mathrm{s}$ and $50 \mathrm{~m}$,

Case I (15 m)

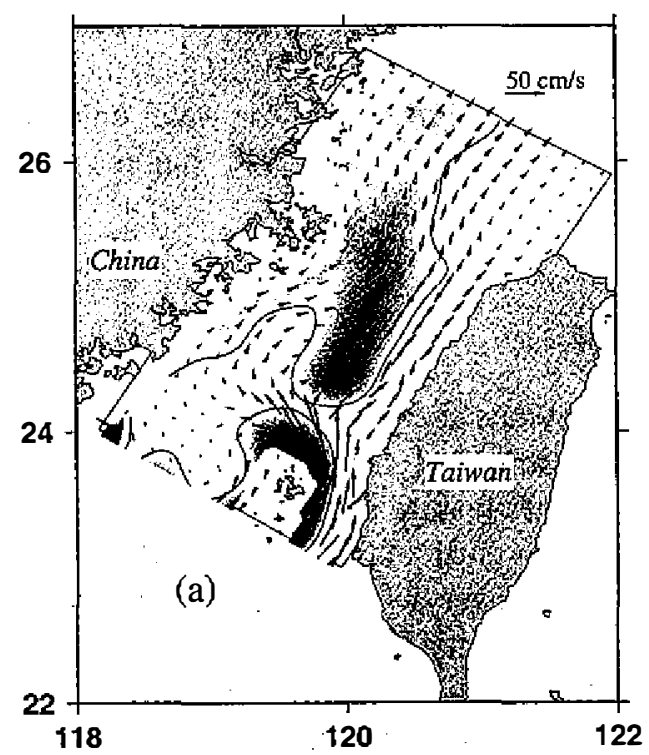

Case II (15 m)

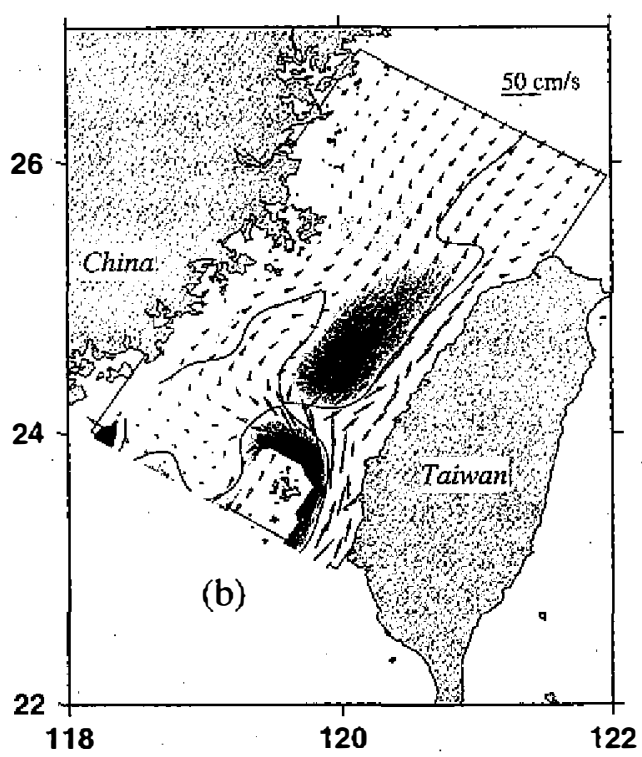

Fig. 12. Horzontal velocity and the vertical component of the relative vorticity distributions at $15 \mathrm{~m}$ for the Case I (a) and Case II (b) on day 250. The solid curve is the contour line of zero relative vorticity and the shaded area denotes the region of posive relative vorticity. 
respectively, and $\mathrm{C}$ is 0.002 in our model. We then have $\mathrm{R}$ as about $10^{-5} \mathrm{~s}^{-1}$. The relative vorticity, generated by the topographic variation, is dampened by the bottom friction as the water is traveling a distance of about $\frac{|\vec{u}|}{R}$, which is about $25 \mathrm{~km}$ in Case I.

Since the horizontal scale of the CYR is about $50 \mathrm{~km}$, we may infer that the negative $\zeta$ acquired by the water column as it approaches the CYR is lost before the water moves to the north of the CYR. Meanwhile, due to the stretching effect, the water column will obtain positive relative vorticity as it flows over the CYR. Hence there is a region of positive vorticity in the middle of the swait to the north of the CYR. The flow pattern associated with this positive vorticity distribution should have a stronger velocity along the eastern bank of the strait, especially in Case II (see Fig. 11). However, when the density gradient in the middle of the strait is strong enough (Case I), the thermal wind relation indicates that the flow should not be weak within the frontal zone. In this case, we may expect a more uniformly distributed velocity profile in the eastern half of the strait, as revealed in Fig. 8.

Figure 13 shows the distribution of velocity and vertical component of the relative vorticity at $15 \mathrm{~m}$ for Case I-1 and II-1 on day 20. The $\varsigma$ distribution of Case II-1, Fig. 13(b), has a similar pattern to that of Case II, Fig. 12(b). However, the flow pattern for Case I-1 becomes very different to that of the Case I. This is due to the fact that the density front in the middle of the strait is in equilibrium with the velocity field in Case $\mathrm{I}$, and this equilibrium is disturbed when the flow rate is decreased in Case I-1. The warm water to the east of the front tends to move westward during the following geostrophic adjustment process. If the density contrast is strong enough, some of the available potential energy associated with the original front will be released. Since the topography to the north of the CYR has a cross-strait slope, Fig. 1, then the westward spreading of the warm water is accompanied by compression of the water column. Therefore the relative vorticity in the middle of the strait north of the CYR changes from positive values in Case I, into negative values in Case I-1.

\section{DISCUSSION AND CONCLUDING REMARKS}

From the numerical model results shown in the previous section, we may infer that the flow in the TS is determined by the interactions of the stratified inflow from the PHC and topographic features like the CYR and the KYD. A distinct feature is the transfer of vertical stratification in the PHC into the horizontal density gradient north of the CYR. A reduction of the transport of the inflow in the PHC decreases the velocity everywhere in the TS through the adjustment in the water level distribution. However the density field is adjusted at a much slower rate, so that the original density gradient is not in equilibrium with the velocity field and some of the available potential energy is released if the density gradient is strong enough. This is a common geostrophic adjustment process, and has been studied quite extensively in a broad area of ocean enviroments, e.g., Tandon and Garrett (1994). The problem in the TS is more complicated, however, due to the fact that the CYR is almost perpendicular to the density front to the north of it; Fig. 6(b). This front is maintained by the convergence of the warm 
Case l-1 (15 m)

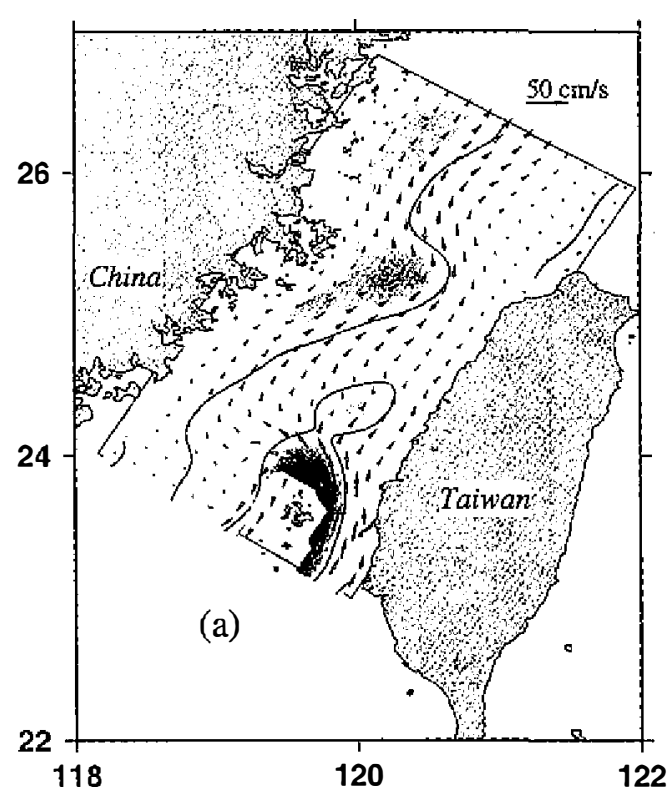

Case II-1 (15 m)

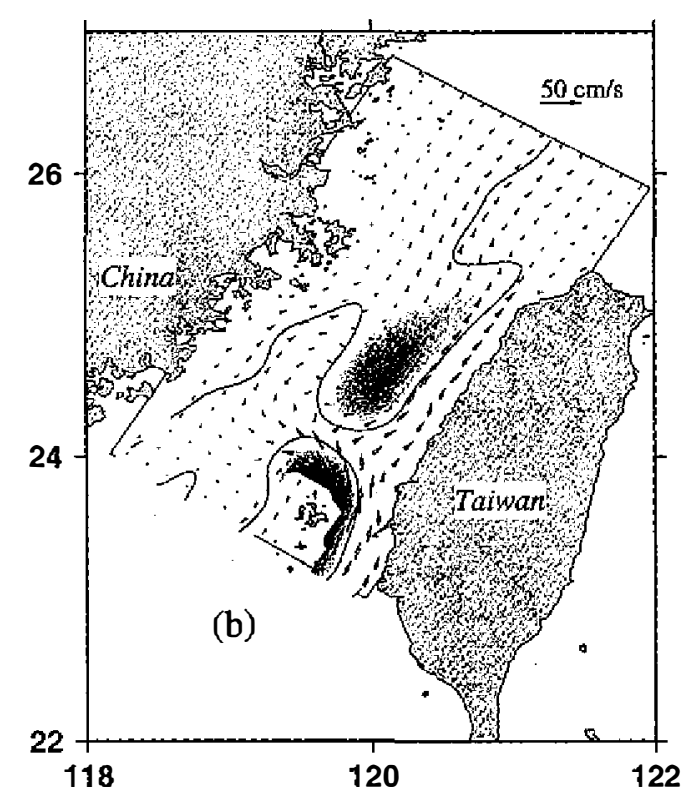

Fig. 13. Horzontal velocity and the vertical component of the relativevorticity distributions at $15 \mathrm{~m}$ for Case I-1 (a) and Case II-1 (b) on day 20. The solid curve is the contour line of zero relative vorticity and the shaded area denotes the region of positive relative vorticity.

water, which crosses over the CYR, and the cold water, which turns around the western edge of the CYR in Case I. During the restratification in Case I-1, the velocity of the cold water turning around the CYR becomes much weaker than the warm water passing over the CYR. Therefore the isotherms follow the depth contours more closely, and the warm water immediately to the north of the CYR moves westward and parallel to the northern edge of the CYR, Fig. 7(b). This cross-strait velocity will cut through the isobath and change the relative vorticity of the flow. The meandering of the current to the north of the CYR in Case I-1 may be attributed to this process.

Figure 7(a) also shows that the meandering of the current in the northern TS is accompanied by an anti-cyclonic eddy in the KYD. This eddy tends to block the northward flow from the TS and pushes it westward. This eddy motion also enhances the westward intrusion of water to the north of Taiwan. Chern and Wang (1992) found that this kind of flow pattern occurs only when the outflow from the TS is weak and this is consistent with the flow condition shown in Fig. 7.

Figures 14 (a) and (b) show the sea surface temperature (SST) contours derived from NOAA 12 and NOAA 14 images on 12 and 17 August 1998, respectively. The isotherms in Fig. 14(a) are roughly parallel to the coast, but in Fig. 14(b) both the westward intrusion of 
(a)

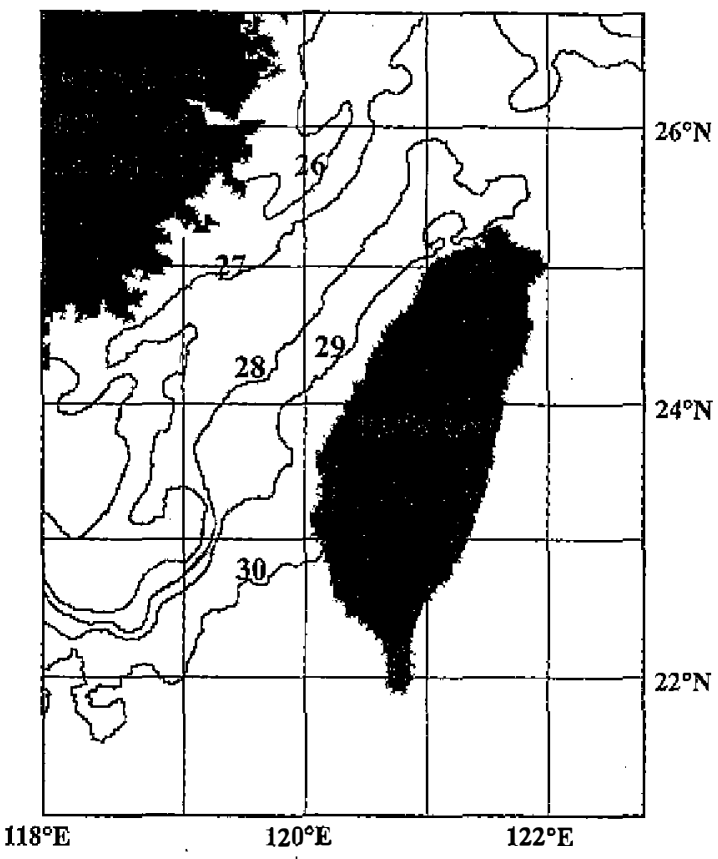

(b)

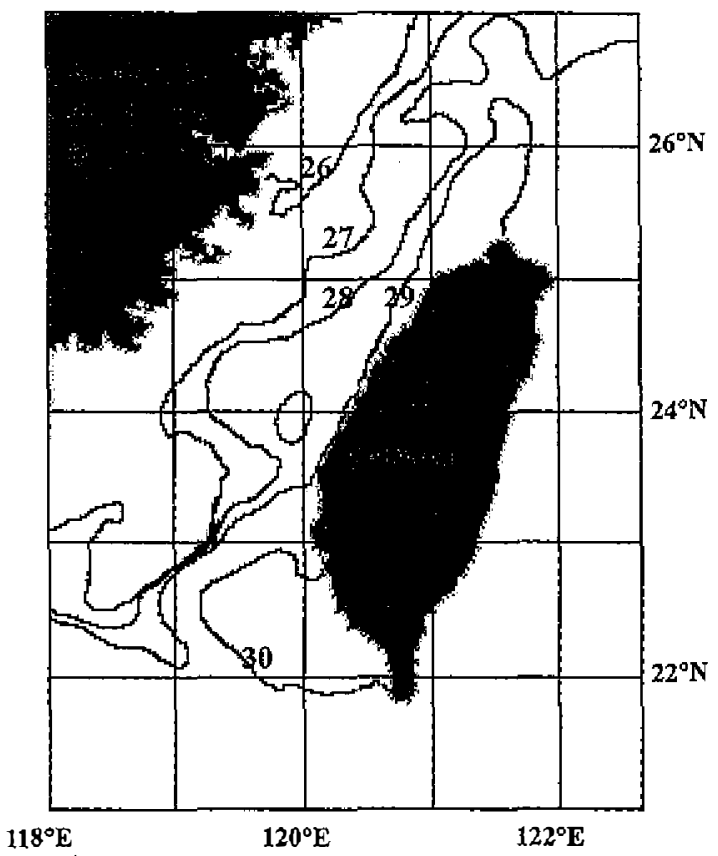

Fig. 14. The sea surface temperature distribution (in ${ }^{\circ} \mathrm{C}$ ) of the TS, derived from the SST images of NOAA 12 on 12 August 1998 (a) and NOAA 14 on 17 August 1998 (b).

warm water $\left(\geq 28^{\circ} \mathrm{C}\right)$ above the CYR, and the eastward movement of cold water $\left(\leq 28^{\circ} \mathrm{C}\right)$ north of the CYR are evident. During 9-11 August 1998, Typhoon Penny occurred in the northern SCS. Hence the winds should have been strong in that area. The stick diagram, Fig. 15, of wind observed at Tungsha (position A in Fig. 3) during 9-17 August 1998 also depicts strong winds during 9-1.1 August, which become calm during 12-17 August. Therefore the image on 12 August corresponds to a larger northward transport in the PHC and the image on 17 August corresponds to a reduced transport event. This inference is consistent with the numerical model results in the previous section.

To summarize, the numerical model studies in this work show that the flow pattern in the TS depends on the stratification and flow rate of the incoming water from the PHC. With a sufficiently strong stratification and volume transport, surface warm water from the PHC can flow over the CYR, and the subsurface cold water tums around the western edge of the CYR. There is a horizontal density gradient to the north of the CYR. A reduction in the flow rate in the PHC decreases the supply of cold water toward the western part of the strait, making maintenance of the original density gradient strength north of the CYR impossible. During the subsequent adjustment process, the current begins to meander and an anticyclonic eddy occurs in the KYD. 


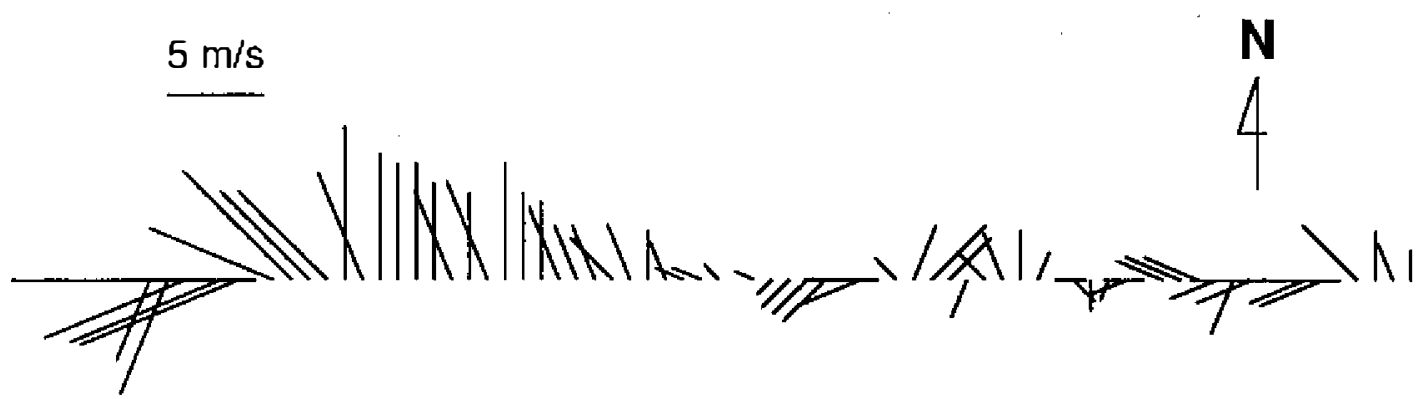

$\begin{array}{lllllllll}9 & 10 & 11 & 12 & 13 & 14 & 15 & 16 & 17\end{array}$

Fig. 15. Stick diagram of wind at Tungsha (Sta. A in Fig. 3) during 9-17 August 1998.

Acknowledgments This work was sponsored by the National Science Council of the Republic of China under Grant NSC 87-2611-M-002A-008-AP7. The satellite SSM/I wind data were obtained from the NASA Physical Oceanography Distributed Active Archive Center at the Jet Propulsion Laboratory, California Institute of Technology.

\section{REFERENCES}

Blumberg, A. F., and G. L. Mellor, 1987: A description of a three-dimensional coastal ocean circulation model. Three-Dimensional Coastal Ocean Models. In: N. S. Heaps (Ed), AGU.

Chao, S. Y., and T. Paluszkiewicz, 1991: The hydraulics of density currents over estuarine sills. J. Geophys. Res., 96, 7065-7076.

Chern, C. S., and J. Wang, 1992: The influence of Taiwan Strait waters on the circulation of the Southem East China Sea. La mer, 30, 223-228.

Fan, K. L., and C. Y. Yu, 1981: A study of water masses in the seas of southernmost Taiwan. Acta Oceanogr. Taiwanica, 12, 94-111.

Jan, S., C. S. Chern, and J. Wang, 1994: A numerical study on the currents in the Taiwan Strait during summertime. La mer, 32, 225-234.

Semtner, A. J., 1986: Finite difference formulation of a world ocean model. Proceedings of the NATO advanced study institute on advanced physical oceanographic numerical modelling In: J. J. O’Brien (Ed.), D. Reidel Publishing Co., Dordrecht, 608 pp.

Tandon, A., and C. Garrett, 1994: Mixed layer restratification due to a horizontal density gradient. J. Phys. Oceanography, 24, 1419-1924.

Wang, J., and C. S. Chern, 1988: On the Kuroshio Branch in the Taiwan Strait during wintertime. Prog. Oceanogr., 21, 469-491.

Wang, J., and C. S. Chern, 1992: On the distribution of bottom cold waters in Taiwan Strait 
during summertime. La mer, 30, 213-221. 